\title{
Отражение от боковой грани кристалла $\mathrm{PbSb}_{2} \mathrm{Te}_{4}$
}

\author{
(C) С.А. Немов ${ }^{1,2,3}$, Ю.В. Улашкевич ${ }^{4}$, М.В. Погумирский ${ }^{5}$, О.С. Степанова ${ }^{2}$ \\ ${ }^{1}$ Санкт-Петербургский политехнический университет им. Петра Великого, \\ 195251 Санкт-Петербург, Россия \\ ${ }^{2}$ Санкт-Петербургский государственный электротехнический университет „ЛЭТИ“ им. В.И. Ульянова (Ленина), \\ 197376 Санкт-Петербург, Россия \\ 3 Забайкальский государственный университет, \\ 672039 Чита, Россия \\ ${ }^{4}$ Физико-технический институт им. А.Ф. Иоффре Российской академии наук, \\ 194021 Санкт-Петербург, Россия \\ ${ }^{5} \mathrm{OOO}$ „ФАРЭКСПОРТ“, \\ 190005 Санкт-Петербург, Россия \\ E-mail: nemov_s@mail.ru
}

Поступила в Редакцию 7 ноября 2019 г.

В окончательной редакции 12 ноября 2019 г.

Принята к публикации 12 ноября 2019 г.

Впервые измерены спектры отражения от боковой грани кристалла $\mathrm{PbSb}_{2} \mathrm{Te}_{4}$ при комнатной температуре в поляризованном свете в диапазоне волновых чисел $50-4000 \mathrm{~cm}^{-1}$. Показана значительная анизотропия кристалла в направлениях вдоль и поперек слоев кристалла. Выявленные особенности спектров интерпретированы вкладом плазменных колебаний и колебаний кристаллической решетки. Рассчитанные значения параметров диэлектрической функции в рамках модели Друде-Лоренца согласуются с экспериментальными данными.

Ключевые слова: термоэлектрические материалы, кристалл $\mathrm{PbSb}_{2} \mathrm{Te}_{4}$, спектр отражения, модель ДрудеЛоренца.

DOI: $10.21883 /$ FTP.2020.03.49023.9308

\section{1. Введение}

В [1,2] были измерены электрофизические параметры тройного кристалла $\mathrm{PbSb}_{2} \mathrm{Te}_{4}$ : коэффициенты Зеебека и Холла, а также удельной электропроводности. Обнаруженная значительная анизотропия свойств обусловлена структурой кристаллов слоистых тетрадемитоподобных халькогенидов, имеющих ромбоэдрическую симметрию, существующих в двух фазах: R32 и R3m [3,4]. В то же время в [5] было отмечено, что малая изученность анизотропии эффективной массы свободных носителей заряда и зонного спектра в кристалле $\mathrm{PbSb}_{2} \mathrm{Te}_{4}$ не позволяет провести детальное сравнение найденных параметров. В связи с высокой концентраций дырок $\left(p \sim 10^{20} \mathrm{~cm}^{-3}\right)$, обусловленной большим количеством разнообразных электрически активных собственных точечных дефектов электронные спектры кристаллов мало изучены. Дополнительную информацию о зонной структуре и параметрах свободных носителей (дырок) можно получить с помощью оптических измерений. В [6] был исследован спектр отражения от грани скола, перпендикулярной тригональной оси кристалла $\mathbf{C}_{\mathbf{3}}$. Оптическими методами был получен ряд электрических параметров, согласующихся с результатами электрофизических методов. Также было показано, что в области волновых чисел $v=50-4000 \mathrm{~cm}^{-1}$ спектр отражения хорошо описывается моделью Друде-Лоренца с использованием плазменных колебаний свободных зарядов и двух решеточных фононов.

Следует отметить, что с помощью оптического исследования спектров отражения от граней сколов кри- сталлов принципиально нельзя получить сведения об анизотропии свойств, поскольку при нормальном падении луча вектор $\mathbf{E}$ всегда перпендикулярен оси $\mathbf{C}_{\mathbf{3}}$ кристалла и, следовательно, получаемый спектр отражения не зависит от ориентации светового потока. Однако большинство оптических измерений полупроводниковых кристаллов, имеющих ось симметрии третьего порядка или выше, выполнено при отражении от грани скола. Это объясняется простотой получения плоскости скола и ее обновления, в отличие от боковой поверхности, получение удовлетворительной информации об оптических свойствах которой требует больших материальных затрат и выбора не всегда обоснованных критериев.

В настоящей работе исследованы спектры отражения от боковой поверхности кристалла $\mathrm{PbSb}_{2} \mathrm{Te}_{4}$ после того, как она была отполирована по специальной методике. В качестве критерия полировки использовалось подобие спектров, полученных от граней сколов и одного из спектров отражения от боковой грани, полученного для поляризованного света, электрический вектор $\mathbf{E}$ которого лежит в плоскости, перпендикулярной тригональной оси кристалла.

\section{2. Методика шлифования и полирования}

Кристалл $\mathrm{PbSb}_{2} \mathrm{Te}_{4}$ закреплялся на стеклянную подложку (далее блок) размером $80 \times 80$ мм, толщиной 8 мм при помощи смолы наклеечной НC-0.5. Свободное пространство на стеклянной подложке заполнялось 
кусочками стекла К8 для обеспечения равномерности обработки грани кристалла. Блок устанавливался на станок LOH HPL-150-2 и обрабатывался методом свободного притира на алмазном инструменте с размером зерна $\sim 30$ мкм. Алмазные зерна на инструменте были закреплены с помощью гальванической связки. В процессе шлифования блок интенсивно омывался водой. Время шлифования грани кристалла составляло 1 мин с нагрузкой в 5 кг. На втором этапе шлифования в качестве инструмента использовались одноразовые подложки, выпускаемые компанией DAC VISION марки DURAFILM 15MIC, размер зерна $\sim 15$ мкм. Время обработки составляло 5 мин с нагрузкой в 5 кг. Общая величина съема за два этапа шлифования составила 0.3 мм. Затем, используя одноразовые подложки, выпускаемые компанией DAC VISION марки PDR 9100L, проводили полировку с использованием водной эмульсии на основе оксида церия марки CE 475 ST. Время полировки 14 мин. Для всех трех этапов обработки использовались следующие режимы настройки станка: скорость вращения 100 об/мин, смещение поводка 20 мм, децентровка поводка 15 мм.

При помощи нагрева блока с кристаллом $\mathrm{PbSb}_{2} \mathrm{Te}_{4}$ до температуры $70^{\circ} \mathrm{C}$ была осуществлена разблокировка, и кристалл отмывался этиловым спиртом. Чистая полированная грань кристалла для защиты заклеивалась пленкой DAC VISION ST-4005. Противоположная грань кристалла $\mathrm{PbSb}_{2} \mathrm{Te}_{4}$ также была заблокирована на стеклянный блок, ее шлифование и полирование было проведено по вышеописанной методике.

Отполированные поверхности граней кристалла $\mathrm{PbSb}_{2} \mathrm{Te}_{4}$ были аттестованы как соответствующие III классу чистоты.

\section{3. Эксперимент}

В настоящей работе исследования были проведены на кристалле $\mathrm{PbSb}_{2} \mathrm{Te}_{4}$, синтезированном по методу Чохральского [7]. Электрические параметры (коэффициент Холла $R_{i k l}$ и концентрация дырок $p$ ) кристалла $\mathrm{PbSb}_{2} \mathrm{Te}_{4}$, а также кристалла, использованного в работе [6], приведены в табл. 1.

В средней инфракрасной (ИК) области (MIR) спектры отражения были измерены с помощью спектрометра $1720 X$ фирмы Perkin Elmer. Для измерения в дальней ИК области (FIR) использовался фурье-спектрометр

Таблица 1. Электрические параметры кристаллов $\mathrm{PbSb}_{2} \mathrm{Te}_{4}$ при $300 \mathrm{~K}$

\begin{tabular}{l|c|c}
\hline $\begin{array}{l}\text { Геометрия } \\
\text { измерений }\end{array}$ & $R_{i k l}, \mathrm{~cm}^{3} /$ Кул & $p, 10^{18} \mathrm{~cm}^{-3}$ \\
\hline $\mathbf{E} \perp \mathbf{C}_{3}[6]$ & 0.028 & 225 \\
$\mathbf{E} \perp \mathbf{C}_{3}[6]$ & 0.038 & 165 \\
$\mathbf{E} \| \mathbf{C}_{\mathbf{3}}$ & 0.02 & 165
\end{tabular}

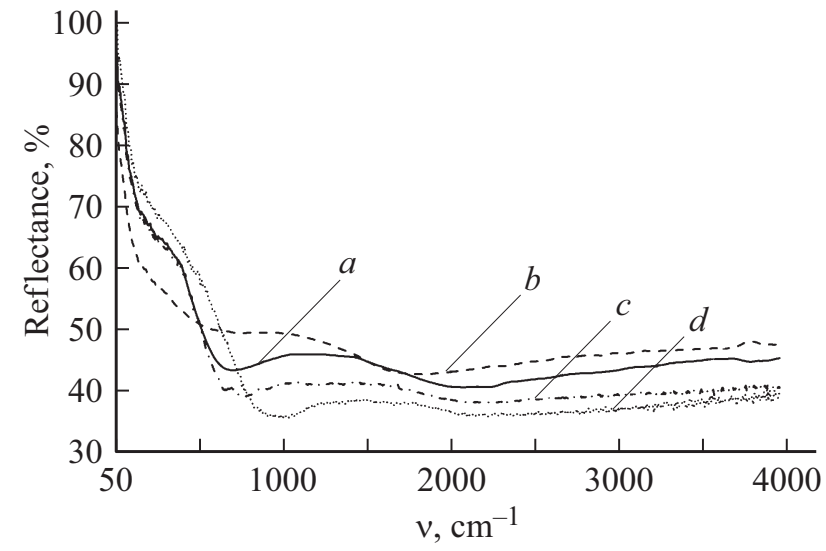

Спектры отражения от кристалла $\mathrm{PbSb}_{2} \mathrm{Te}_{4}$ : от боковой грани, вектор $\mathbf{E} \perp \mathbf{C}_{\mathbf{3}}(a)$, вектор $\mathbf{E} \| \mathbf{C}_{3}(b)$, от грани скола $(c)$, из работы $[6](d)$.

IFS-113v фирмы Bruker. Спектральное разрешение приборов составляло $2 \mathrm{~cm}^{-1}$. Измерение спектров в поляризованном свете проводилось с помощью поляризаторов: в среднем диапазоне с помощью поляризатора KRS, в дальнем ИК диапазоне использовался полиэтиленовый поляризатор. Измерение спектров проводили несколько раз, спектры усредняли и „сшивали“ друг с другом в области перекрывания FIR и MIR. Полученные таким образом спектры отражения приведены на рисунке. Здесь же для сравнения приведен спектр отражения из [6].

\section{4. Обсуждение результатов}

Из рисунка можно видеть, что все представленные спектры отражения от граней кристаллов $\mathrm{PbSb}_{2} \mathrm{Te}_{4}$ имеют два минимума. Эти же два минимума наблюдаются в спектре легированного медью образца [8], a также в кристаллах $\mathrm{Bi}_{2} \mathrm{Te}_{3}$, как в чистых, так и слабо легированных [9-11], при измерении отражения в достаточно широкой области. Интересно отметить, что в кристаллах, имеющих схожую симметрию, таких как, например, $\mathrm{Bi}_{2} \mathrm{Se}_{3}$ или $\mathrm{Sb}_{2} \mathrm{Te}_{3}$, в спектрах отражения второго минимума не наблюдается [12]. В работе [10] была предпринята попытка объяснить наличие второго минимума, связав его с предшествующим подъемом отражения за счет электрон-плазмонного взаимодействия. Однако в [9] такое объяснение было поставлено под сомнение из-за большого различия в энергиях процессов, участвующих во взаимодействии. Поэтому природа явления пока не разгадана, хотя математически эффект второго минимума описывается довольно точно $[6,9]$.

Спектры отражения от грани скола и от боковой поверхности при падении поляризованного света с вектором $\mathbf{E}$, перпендикулярным тригональной оси кристалла, являются подобными. Это может рассматриваться как свидетельство хорошей полировки боковых граней, описанной выше. Спектр отражения поляризованного света с вектором $\mathbf{E}$, параллельным оси $\mathbf{C}_{3}$, отличается 
Таблица 2. Параметры аппроксимации экспериментальных спектров моделью Друде-Лоренца

\begin{tabular}{c|c|c|c|c}
\hline Параметр & {$[6]$} & $\begin{array}{c}\text { Грань } \\
\text { скола }\end{array}$ & $\begin{array}{c}\text { Боковая грань, } \\
\mathbf{E} \perp \mathbf{C}_{\mathbf{3}}\end{array}$ & $\begin{array}{c}\text { Боковая грань, } \\
\mathbf{E} \| \mathbf{C}_{\mathbf{3}}\end{array}$ \\
\hline$\varepsilon_{\infty}$ & 19.9 & 21.5 & 26.3 & 29.2 \\
$v_{t}, \mathrm{~cm}^{-1}$ & 331 & 328 & 328 & 425 \\
$v_{t 1}, \mathrm{~cm}^{-1}$ & 1872 & 1708 & 1560 & 1345 \\
$S, 10^{6} \mathrm{~cm}^{-2}$ & 13.4 & 7.9 & 8.9 & 8.3 \\
$S_{1}, 10^{6} \mathrm{~cm}^{-2}$ & 8.6 & 13.5 & 12.4 & 10.1 \\
$\gamma, \mathrm{cm}^{-1}$ & 723 & 523 & 547 & 908 \\
$\gamma_{1}, \mathrm{~cm}^{-1}$ & 1075 & 1708 & 913 & 761 \\
$v_{p}, \mathrm{~cm}^{-1}$ & 333 & 296 & 277 & 211 \\
$\omega_{p}, 10^{14} \mathrm{c}^{-1}$ & 0.63 & 0.56 & 0.52 & 0.40 \\
$\gamma_{p}, \mathrm{~cm}^{-1}$ & 14.1 & 50 & 44 & 94 \\
$\chi^{2}$ & 0.33 & 0.24 & 0.46 & 0.39 \\
$\sigma_{\mathrm{opt}}$, & 2610 & 627 & 766 & 231 \\
$\mathrm{OM}^{-1} \cdot \mathrm{cm}^{-1}$ & & & &
\end{tabular}

Примечание. $\chi^{2}-$ среднеквадратичная невязка аппроксимации.

от первых двух как по интенсивности минимумов, так и по их положению. Это однозначно указывает на существование показанной в $[1,2]$ анизотропии электрических свойств кристаллов $\mathrm{PbSb}_{2} \mathrm{Te}_{4}$.

Как видно из рисунка, спектр отражения от боковой грани имеет те же характерные особенности, что и спектр отражения от грани скола, поэтому для моделирования спектров и расчета параметров кристаллов так же, как и в [6], может быть использована диэлектрическая функция [6].

Диэлектрическая функция учитывает вклады плазменных колебаний дырок и колебаний кристаллической решетки, описываемых в модели осцилляторов [6]:

$$
\begin{aligned}
\varepsilon(v) & =\varepsilon_{1}(v)+i \varepsilon_{2}(v) \\
& =\varepsilon_{\infty}+\frac{v_{p}^{2} \varepsilon_{\infty}}{-v^{2}+i \nu \Gamma}+\sum_{i}^{n} \frac{S_{i}}{v_{t i}^{2}-v^{2}+i v \gamma_{i}},
\end{aligned}
$$

где $\varepsilon_{1}$ и $\varepsilon_{2}-$ вещественная и мнимая составляющие диэлектрической проницаемости, $\varepsilon_{\infty}-$ высокочастотная (на оптических частотах) диэлектрическая проницаемость, $v_{t i}$ - волновые числа осцилляторных колебаний, $S_{i}$ - амплитуды осцилляторных колебаний, $\gamma_{i}$ - демпфирование осцилляторов, $v_{p}$ - плазменное волновое число (частота $\omega_{p}=2 \pi c v_{p}$ ), $\Gamma-$ демпфирование плазмы (время релаксации $\left.\tau_{p}=1 / 2 \pi c \Gamma\right), c$ скорость света.

Стандартным методом была выполнена аппроксимация экспериментальных спектров моделью ДрудеЛоренца. Было отмечено, что для приемлемого согласования с экспериментом достаточно учитывать вклад от колебаний двух типов осцилляторов и свободной плазмы. Параметры аппроксимации представлены в табл. 2.

В последней строке табл. 2 приведены значения удельной электропроводности $\sigma_{\mathrm{opt}}$, определенные оптическим методом [12]. Из этих данных можно видеть значительную анизотропию электрических свойств, что объясняется различной подвижностью дырок вдоль и поперек слоев кристалла. Из спектров отражения можно также получить оценку эффективной массы дырок, но получаемые при этом значения оказываются завышенными. Как уже отмечалось в [5], это связано со сложным строением валентной зоны рассматриваемого тройного кристалла и с существованием при комнатной температуре двух типов дырок.

\section{5. Заключение}

С помощью специальной методики отполированы боковые грани слоистого кристалла $\mathrm{PbSb}_{2} \mathrm{Te}_{4}$. Измерены спектры отражения при комнатной температуре в поляризованном свете в диапазоне $50-4000 \mathrm{~cm}^{-1}$. Наблюдаемые особенности спектров могут быть связаны с вкладом плазменных колебаний и колебаний кристаллической решетки. В рамках модели Друде-Лоренца рассчитаны параметры (плазменная частота, вещественная и мнимая составляющие диэлектрической проницаемости, высокочастотная диэлектрическая проницаемость и др.), значения которых согласуются с экспериментальными данными. Показана значительная анизотропия кристалла в направлениях вдоль и поперек слоев кристалла.

\section{Конфликт интересов}

Авторы заявляют, что у них нет конфликта интересов.

\section{Список литературы}

[1] М.К. Житинская, С.А. Немов, Л.Е. Шелимова, Т.Е. Свечникова, П.П. Константинов. ФТТ, 50, 8 (2008).

[2] Н.М. Благих. Автореф. канд. дис. (СПб., СПбПУ, 2013).

[3] Л.Е. Щелимова, О.Г. Карпинский, Т.Е. Свечникова, Е.С. Авилов, М.А. Кретова, В.С. Земсков. Неорг. матер., 40, 1440 (2004).

[4] С.А. Немов, Н.М. Благих, В.Д. Андреева. Научн. ведомости Белгородского ун-та. Сер.: Математика. Физика, 11 (154), вып. 31 (2013).

[5] С.А. Немов, Н.М. Благих, Л.Е. Шелимова. ФТП, 47, 18 (2013).

[6] С.А. Немов, Ю.В. Улашкевич, А.В. Поволоцкий, И.И. Хламов. ФТП, 50, 1343 (2016).

[7] С.А. Немов, Ю.В. Улашкевич. ФТП, 51, 3 (2017).

[8] С.А. Немов, Ю.В. Улашкевич, А.А. Аллаххах. ФТП, 51, 10 (2017).

[9] Н.П. Степанов, А.А. Калашников, Ю.В. Улашкевич. Опт. и спектр., 109, 6 (2010).

[10] M. Stordeur, M. Stolzer, H. Sobotta, V. Riede. Phys. Status Solidi B, 150, 1 (1988).

[11] S.V. Dordevic, M.S. Wolf, N. Stojilovic, Hechang Lei, C. Petrovic. J. Phys.: Condens. Matter, 25, 7 (2013).

[12] Ю.И. Уханов. Оптические свойства полупроводников (М., Наука, 1977).

Редактор Л.В. Шаронова 


\section{Reflection from side face of $\mathrm{PbSb}_{2} \mathrm{Te}_{4}$} crystal

S.A. Nemov ${ }^{1,2,3}$, Yu.V. Ulashkevivh ${ }^{4}$, M.V. Pogumirsky ${ }^{5}$, O.S. Stepanova ${ }^{2}$

${ }^{1}$ Peter the Great St. Petersburg Polytechnic University, 195251 St. Petersburg, Russia

2 Saint Petersburg Electrotechnical University "LETl“, 197376 St. Petersburg, Russia

${ }^{3}$ Transbaikal State University, 672039 Chita, Russia

${ }^{4}$ Ioffe Institute, 194021 St. Petersburg, Russia

${ }^{5}$ LLC „FAREXPORT“, 190005 St. Petersburg, Russia

Abstract The reflection spectra from the side face of a $\mathrm{PbSb}_{2} \mathrm{Te}_{4}$ crystal were measured at the room temperature in polarized light in the range $50-4000 \mathrm{~cm}^{-1}$. Significant anisotropy of the crystal was revealed in the directions along and across the crystal layers. The revealed spectral features are interpreted as connected width the contribution of plasma oscillations and crystal lattice vibrations. The calculated dielectric function parameters in the framework of the Drude-Lorentz model are consistent with the experimental data. 